\title{
Análisis Estadístico de Movilidad y Educación Vial dentro de la Escuela Superior Politécnica de Chimborazo
}

Statistical Analysis of Mobility and Road Education within the Polytechnic

\author{
School of Chimborazo
}

Fabian Patricio Londo Yachambáy. ${ }^{1}$ \& Narcisa de Jesús Sánchez Salcán. ${ }^{2}$

\begin{abstract}
The Campus of the Polytechnic School of Chimborazo (ESPOCH) has an area of 119.5 hectares and its civil infrastructure covers 13.53 hectares, so its road infrastructure has a total extension of $19.20 \mathrm{~km}$, by these roads Approximately 7500 vehicles circulate daily and as a result of this several mobility problems have arisen.
\end{abstract}

The transport systems that circulate within the ESPOCH are very diverse, private vehicles, taxis and also the inter-institutional bus enter, each of these travels throughout the polytechnic campus, as for the bus its frequency is inadequate for the supply of the polytechnic community Due to the demand of the student sector, taxis have become the mass transport system within the polytechnic due to the large influx of the student sector, especially during academic periods.

The road infrastructure of the ESPOCH has accessible roads for the different means of transport used within it such as: taxis, private and private vehicles, bicycles, among others, it can also be seen that there are adapted ramps in different parts of the campus

1 Escuela Superior Politécnica de Chimborazo, Facultad de administración de Empresas, Ecuador, flondo@espoch.edu.ec

${ }^{2}$ Universidad Nacional de Chimborazo, Facultad de Ciencias de la Educación, Humanas y Tecnologías, , nsanchez@unach.edu.ec 
for the best Accessibility of people with disabilities who must and need to move to various sites, which do not have a relevant signaling for viewing and use of them. In certain circumstances there has also been evidence of disrespect by drivers of vehicles towards pedestrians, where a total lack of Road Education can be clearly noticed.

Adding to all these inconveniences, it can also be mentioned that there is an insufficient parking lot and a bad location within the institution, so it does not provide for the entire automotive fleet that enters. Therefore, a Mobility Plan is necessary for the Chimborazo Polytechnic School campus and must be accompanied by training and awareness on Road Education to administrative staff, teachers and students

Keywords: Mobility, Road Education, Plan

\section{Resumen}

El Campus de la Escuela Superior Politécnica de Chimborazo (ESPOCH) tiene una extensión de 119,5 hectáreas y su infraestructura civil abarca las 13,53 hectáreas, por lo que su infraestructura vial tiene una extensión total de 19,20 km, por estas vías circulan 7500 vehículos diarios aproximadamente y producto de esto ha surgido varios inconvenientes de movilidad.

Los sistemas de transporte que circulan dentro de la ESPOCH es muy diverso, ingresan vehículos particulares, taxis y además el bus interinstitucional, cada uno de estos recorren todo el campus politécnico, en cuanto al bus su frecuencia es inadecuada para el abastecimiento de la comunidad politécnica por la demanda del sector estudiantil, los taxis se han convertido en el sistema de transporte masivo dentro de la politécnica por la gran afluencia del sector estudiantil especialmente en periodos académicos

La infraestructura vial de la ESPOCH cuenta con vías accesibles para los diferentes medios de transporte utilizados dentro de la misma como son: taxis, vehículos privados y particulares, bicicletas entre otros, también puede observarse que existen rampas adaptadas en diferentes partes del campus para la mejor accesibilidad de personas con discapacidad que debe y necesitan desplazarse a diversos sitios, las cuales no cuentan con una señalización pertinente para su visualización y el uso de las mismas. En determinadas circunstancias también se ha evidenciado el irrespeto por parte de los conductores de vehículos hacia los peatones, donde se puede notar claramente un desconocimiento total de Educación Vial.

Sumándose a todos estos inconvenientes también se puede mencionar que existen una insuficiencia de parqueaderos y mala ubicación dentro de la institución, por lo que no abastece para todo el parque automotor que ingresa. Ante lo cual es necesario una Plan de Movilidad al campus de Escuela Superior Politécnica de Chimborazo y que debe ir acompañado de una capacitación y concientización sobre Educación Vial al personal administrativo, docentes y estudiantes. 
Palabras claves: Movilidad, Educación Vial, Plan

\section{Introducción}

Una de las problemáticas a nivel mundial son los accidentes de tránsito, los cuales han sido generados, en gran parte de los casos por malas maniobras por parte de los conductores, por otra parte los peatones también han incido en esta problemática su desconocimiento e irrespeto a las señales de tránsito, esto ha conllevado a pérdidas económicas y humanas, las mismas que se ven reflejadas en el dolor, la consternación y una mala experiencia para quienes sufren y son víctimas de estos casos dolorosos. (Villarroel, 2018)

La educación vial tanto en conductores como peatones es muy importante para la seguridad de los mismo, es una responsabilidad que engloba a toda la sociedad ya que son participes de la movilidad diaria (Mercham, 2011), para esto las autoridades, especialmente los gobiernos de cada ciudad, provincia deben ser los entes encargados de tomar decisiones importantes en esta temática ciudadana para así juntos poder realizar un mejor desempeño en las vías y dentro de un vehículo, teniendo en cuenta que se debe formar, educar a las personas con valores éticos y sobre todo una Educación vial integra, para estos casos se debe mantener una relación colaborativa con diferentes instituciones públicas como lo son: Ministerio de Transporte y Obras Públicas, Ministerio de Salud Pública, Ministerio del Interior, Ministerio de Educación, Policía Nacional del Ecuador, FONSAT, entre otros. Todas estas instituciones tienen propósito en común como es proteger la vida e integridad de todos los usuarios de transporte terrestre en el territorio ecuatoriano.

La Escuela Superior Politécnica de Chimborazo en sus instalaciones cuenta con vías, parqueaderos y servicio de transporte interno, así como existen instituciones que regulan el tránsito en el país, la ESPOCH cuenta con diferentes departamentos para su control como son: departamento de mantenimiento y desarrollo físico, departamento de movilidad y transporte y personal de seguridad, que en conjunto deben asegurar una adecuada movilidad dentro de la ESPOCH.

Por otra parte, se considera que la movilidad es de suma importancia en cualquier institución de educación superior por el alumnado que existe dentro de las mismas y que se debe salvaguardar la integridad física de las mimas.

\section{Metodología}

La presente investigación se realizó a partir de un enfoque cuantitativo, posee un carácter descriptivo porque se observó, registró y describió la problemática de movilidad en la ESPOCH.

Se desarrolló dentro del paradigma de la investigación - acción, pues ella permite analizar situaciones vividas por parte de los miembros de la comunidad universitaria y avanzar en la construcción de soluciones a las necesidades presentadas en temas de Movilidad y Educación Vial.

Kemmis \& Taggart (como se citó en Benjumeda, 2012), el proceso puede resumirse en las siguientes fases:

- Diagnóstico y reconocimiento de las situación actual

- Desarrollo de un plan de acción, críticamente informado, para mejorar aquello que ya está ocurriendo 
- Actuación para poner el plan en práctica y la observación de sus efectos en el contexto que tiene lugar la reflexión en torno a los efectos como base para una nuevo plan de movilidad dentro del campus.

El diseño de la investigación es no experimental, porque el estudio se realizó sin manipular deliberadamente las variables.

Como nos indica Mateo (2000), las metodologías ex-post-facto o no experimental son las más utilizadas en el ámbito educativo, proporcionándonos técnicas para describir la realidad, analizar relaciones, categorizar, simplificar y organizar las variables que configuran el objeto de estudio.

El diagrama simbólico es: (Sánchez-Reyes: 62)

En este caso el tipo de investigación a utilizarse será la investigación descriptiva ya que se va a determinar a través de medidas exploratorias la situación en la que se encuentra la ESPOCH, es decir se empleara un formulario de encuesta para determinar causas consecuencias y problemáticas que se presentan en la movilidad dentro de la institución.

La población de la Escuela Superior Politécnica de Chimborazo, Campus Riobamba es de un total de 14536 estudiantes.

Tabla No1. Población

\begin{tabular}{ll}
\hline POBLACION POR FACULTADES & \\
\hline FACULTADES & TOTAL \\
Administración de Empresas & 2950 \\
Ciencias & 2655 \\
Ciencias Pecuarias & 973 \\
Informática y Electrónica & 1738 \\
Mecánica & 2375 \\
Recursos Naturales & 1155 \\
Salud Pública & 2690 \\
Total & 14536 \\
\hline
\end{tabular}

\section{Muestra.}

Como la población con la que contamos es muy extensa, se procede a emplear una muestra estadística, para lo cual aplicaremos la siguiente formula que se aplica cuado se conoce la población o esta es finita: (Sarai, 2005)

$$
\begin{gathered}
n=\frac{N z^{2} p q}{(N-1) e^{2}+z^{2} p q} \\
n=\frac{(14536)(1.96)^{2}(0.5)(0.5)}{(14536-1)(0.05)^{2}+(1.96)^{2}(0.5)(0.5)}
\end{gathered}
$$




$$
n=374
$$

Calculo de la Fracción Muestral

Para saber distribuir equitativamente la muestra se procede a realizar el cálculo de la fracción muestral: (Sarahi, 2005)

$$
\begin{gathered}
f=\frac{n}{N} \\
f=\frac{374}{14536} \\
f=0.025729224
\end{gathered}
$$

\section{Métodos, técnicas e instrumentos}

En la siguiente investigación utilizaremos los siguientes métodos: Inductivo-Deductivo. Las técnicas que se utilizaran en esta investigación es el de encuesta. Para la encuesta se requiere de un cuestionario que está dirigido para estudiantes, personal administrativo y servicio de la ESPOCH, constando de 12 ítems, las mismas que estarán conformadas por preguntas abiertas, cerradas, de selección múltiple. (Ruiz, 2007)

\section{Resultados.}

La discusión de resultados se realizará en función de los estratos tomados por las diversas facultades que constituyen la ESPOCH, seguidamente se detalla cada ítem con su respectivo resumen y gráfico.

¿Qué tipo de medio de Transporte utiliza para movilizarse dentro de la ESPOCH?

Tabla No2. Medios de trasporte

\begin{tabular}{ll}
\hline Respuesta & N- de Personas \\
\hline Bus Institucional & 40 \\
Auto Particular & 28 \\
Bicicleta & 16 \\
Taxi & 40 \\
Moto & 15 \\
Caminando & 235 \\
Total & $\mathbf{3 7 4}$ \\
\hline
\end{tabular}


Gráfico No1. Medios de transporte utilizado para movilizarse dentro de la ESPOCH
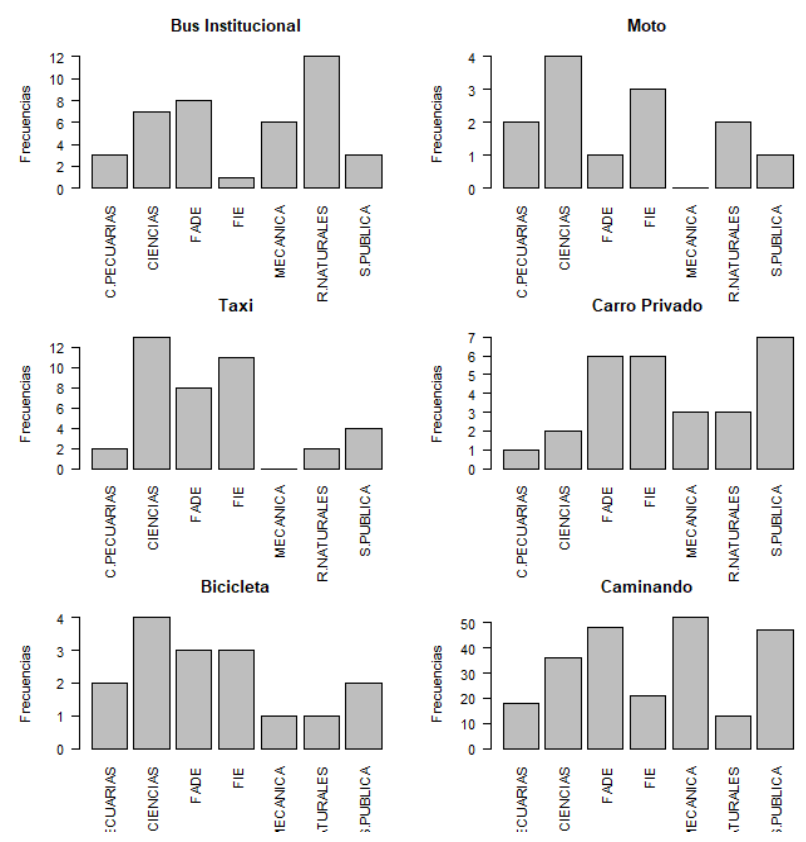

El $63 \%$ de la población a quien se realizó la encuesta respondió que su modo de transportarse dentro de la ESPOCH es ir caminando. El 11\% de estudiantes toman la opción del bus institucional y taxi para movilizarse dentro de la institución. El 7\% hace uso del carro privado para su ingreso a la escuela superior. Existe un $4 \%$ que optan por usar moto y bicicleta para el acceso a la ESPOCH

Gráfico No2. Uso de Taxi
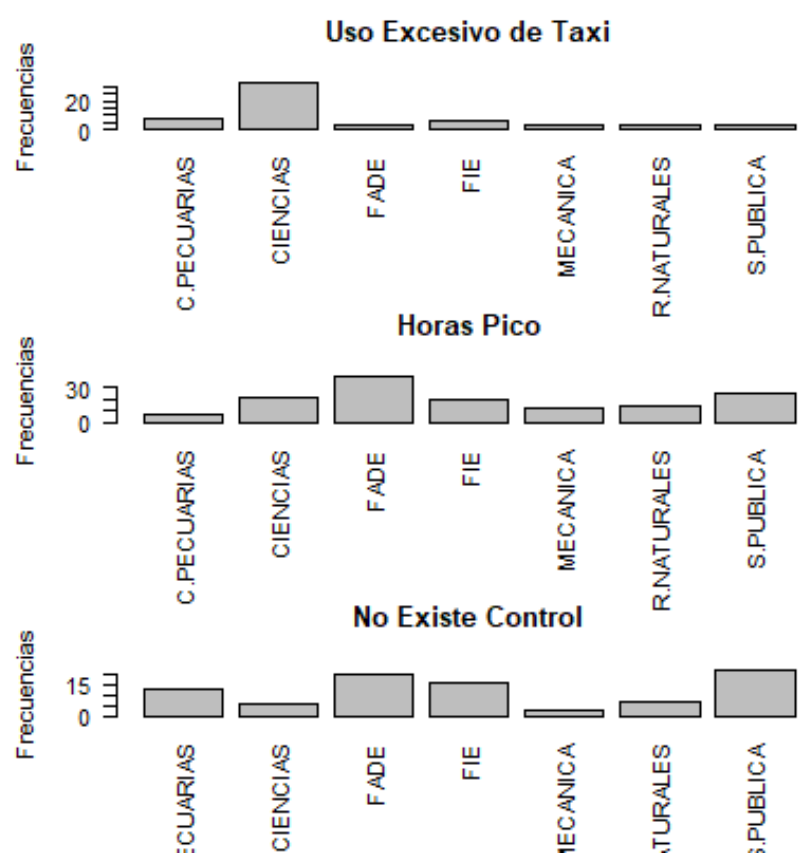
El 52\% de la población piensa que el tráfico dentro de las ESPOCH existe solamente en horas pico. El 29\% de estudiantes hacen referencia que debería existir más control para el ingreso a la institución. El 18\% del personal que existe dentro de la ESPOCH nos dice que la causa para que exista congestión vehicular es el uso masivo de taxis que hacen el ingreso a la institución.

Gráfico No3. Piensa usted que el intervalo de tiempo del bus institucional satisface a las necesidades

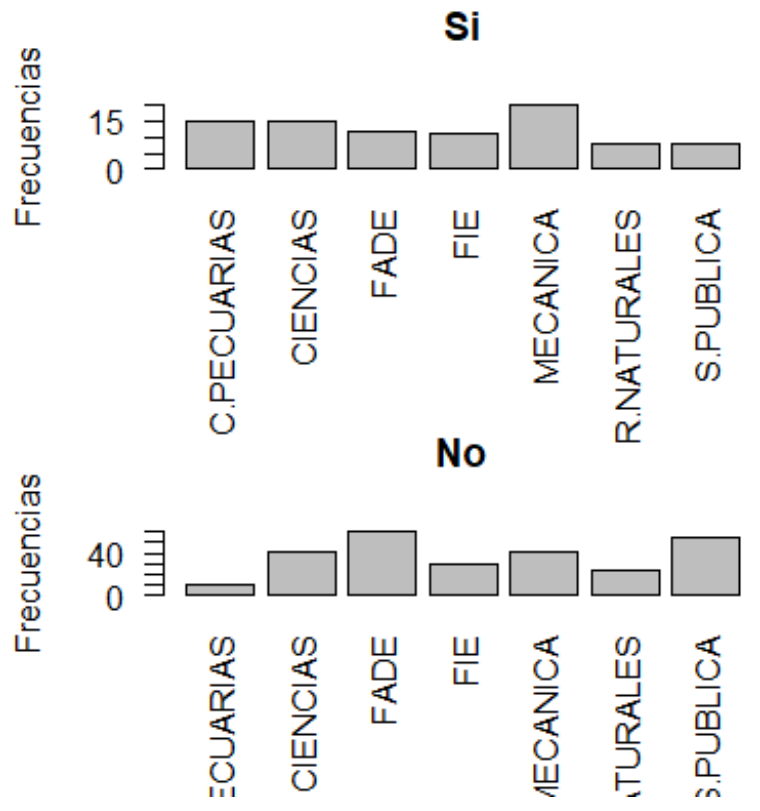

Tabla No3. En qué horario usted ingresa a la ESPOCH

\begin{tabular}{|c|c|c|c|c|}
\hline FACULTAD & MAÑANA & TARDE & NOCHE & \\
\hline FADE & 71 & 20 & 3 & \\
\hline FIE & 32 & 21 & 2 & \\
\hline MECANICA & 37 & 18 & 7 & \\
\hline $\begin{array}{l}\text { CIENCIAS } \\
\text { RECURSOS }\end{array}$ & 33 & 12 & 2 & \\
\hline NATURALES & 30 & 7 & 0 & \\
\hline $\begin{array}{l}\text { SALUD PUBLICA } \\
\text { CIENCIAS }\end{array}$ & 25 & 18 & 8 & \\
\hline PECUARIAS & 27 & 1 & 0 & TOTAL \\
\hline TOTAL & 255 & 97 & 22 & 374 \\
\hline PORCENTAJE & $68 \%$ & $26 \%$ & $6 \%$ & $100 \%$ \\
\hline
\end{tabular}

El 68\% de estudiantes tiene su ingreso desde muy temprano a la institución para sus labores académicas. El $26 \%$ de la población en cambio hacen su arribo en horas de la tarde. Un $6 \%$ de la población ingresa al establecimiento en horas de la noche. 
Tabla No4. Cree que la ESPOCH cuenta con espacios de señalética para movilizarse con bicicleta

\begin{tabular}{lcccc}
\hline FACULTAD & SI & \multicolumn{2}{c}{ NO } & \\
FADE & 12 & 60 & \\
FIE & 11 & 30 & \\
MECANICA & & 20 & 60 & \\
CIENCIAS & & 15 & 45 & \\
RECURSOS & & & \\
NATURALES & 8 & 24 & \\
SALUD PUBLICA & 8 & 55 & \\
CIENCIAS & & & \\
PECUARIAS & 15 & 11 & TOTAL \\
TOTAL & 89 & 285 & 374 \\
\hline
\end{tabular}

El 76\% de estudiantes encuestados piensan que la ESPOCH no cuenta con la señalética necesaria para su desplazamiento en bicicleta dentro de la misma. El 24\% de encuestados recalcan que el establecimiento no cuenta con la señalética adecuada y necesaria para el uso de bicicletas.

Tabla No 5. Con qué frecuencia utiliza el bus institucional

\begin{tabular}{lrrrr}
\hline FACULTAD & SIEMPRE & A VECES & \multicolumn{1}{l}{ NUNCA } & \\
FADE & 1 & 30 & 40 & \\
FIE & 5 & 6 & 70 & \\
MECANICA & 2 & 7 & 40 & \\
CIENCIAS & 7 & 35 & 9 & \\
RECURSOS & & & & \\
NATURALES & 7 & 19 & 7 & \\
SALUD PUBLICA & 3 & 33 & 24 & \\
CIENCIAS & 3 & 23 & 2 & TOTAL \\
PECUARIAS & 28 & 153 & 192 & 373 \\
TOTAL & $8 \%$ & $41 \%$ & $51 \%$ & $100 \%$ \\
PORCENTAJE & & & & \\
\hline
\end{tabular}

El 51\% de estudiantes nunca han utilizado el bus institucional. El 41\% de la población encuestada refleja que en pocas ocasiones hacen uso del bus institucional. El 8\% del personal de la ESPOCH recalca que siempre hacen uso del bus institucional. 
Tabla No6. Que ideas implementaría usted para mejorar la movilidad en ESPOCH

\begin{tabular}{|c|c|c|c|c|c|c|}
\hline FACULTAD & $\begin{array}{l}\text { CICLOVI } \\
\text { A }\end{array}$ & $\begin{array}{l}\text { MAS } \\
\text { SEÑALETIC } \\
\text { A }\end{array}$ & $\begin{array}{l}\text { MAS } \\
\text { BUSE } \\
\text { S } \\
\end{array}$ & $\begin{array}{l}\text { MEJORAMIENT } \\
\text { O DE LAS VIAS } \\
\end{array}$ & $\mathbf{S} / \mathbf{N}$ & $\begin{array}{l}\text { TOTA } \\
\text { L }\end{array}$ \\
\hline FADE & 6 & 24 & 14 & 15 & 15 & 74 \\
\hline FIE & 5 & 18 & 7 & 10 & 5 & 45 \\
\hline MECANICA & 10 & 24 & 10 & 8 & 11 & 63 \\
\hline CIENCIAS & 7 & 20 & 19 & 17 & 4 & 67 \\
\hline RECURSOS & & & & & & \\
\hline $\begin{array}{l}\text { NATURALES } \\
\text { SALUD }\end{array}$ & 0 & 4 & 16 & 3 & 10 & 33 \\
\hline PUBLICA & 7 & 24 & 18 & 1 & 14 & 64 \\
\hline CIENCIAS & 2 & 13 & 12 & 0 & 1 & 28 \\
\hline $\begin{array}{l}\text { TOTAL } \\
\text { PORCENTAJ }\end{array}$ & 37 & 127 & 96 & 54 & 60 & 374 \\
\hline $\mathbf{E}$ & $10 \%$ & $34 \%$ & $26 \%$ & $14 \%$ & $16 \%$ & $100 \%$ \\
\hline
\end{tabular}

El 34\% de estudiantes piden que implemente más señalética en el campus universitario. El 26\% de la población piensan que el establecimiento debe aumentar su flota de buses para su uso y beneficiar así a los mismos. El 16\% no cuentan con ideas pertinentes para el mejoramiento de la movilidad. El $14 \%$ piden que para el mejoramiento de la movilidad empiecen por mejoras las vías y el asfaltado que existe dentro la institución. El 10\% piensan que se debe cuidar el medio ambiente ante lo cual piden la creación de una ruta de ciclovía y de esta forma mejorar la movilidad dentro de la ESPOCH.

Tabla No7. Cree que el bus institucional es accesible para todas las Escuelas de la ESPOCH.

\begin{tabular}{lrrrrr}
\hline \multicolumn{1}{c}{ FACULTAD } & BUENO & REGULAR & MALO & S/N & TOTAL \\
\hline FADE & 15 & 44 & 10 & 5 & 74 \\
FIE & 18 & 20 & 7 & 0 & 45 \\
MECANICA & 9 & 40 & 13 & 1 & 63 \\
CIENCIAS & 2 & 40 & 22 & 3 & 67 \\
RECURSOS & & & & & \\
NATURALES & 4 & 26 & 3 & 0 & 33 \\
SALUD & & & & & \\
PUBLICA & 14 & 31 & 19 & 0 & 64 \\
CIENCIAS & 2 & 8 & 18 & 0 & 28 \\
TOTAL & $\mathbf{6 4}$ & $\mathbf{2 0 9}$ & $\mathbf{9 2}$ & $\mathbf{9}$ & $\mathbf{3 7 4}$ \\
PORCENTAJE & $17 \%$ & $56 \%$ & $25 \%$ & $2 \%$ & $100 \%$ \\
\hline
\end{tabular}

\section{Análisis:}

El 56\% de la población encuestada nos dice que la accesibilidad del bus institucional a diferentes puntos de la ESPOCH es regular. El 25\% de estudiantes nos recalca que el funcionamiento del bus institucional es malo porque que no abastece a todos los lugares 
de la institución. El 17\% cree que la accesibilidad del bus es buena porque si llega a todos los puntos necesarios o puntos clave para el traslado dentro de la misma. El 2\% no cuenta con una respuesta y desconocen el funcionamiento del bus institucional.

Tabla No8. Cuál es su apreciación acerca de la movilidad de transporte de la ESPOCH

\begin{tabular}{lrrrrr}
\hline \multicolumn{1}{r}{ FACULTAD } & BUENO & REGULAR & MALO & S/N & TOTAL \\
\hline FADE & 10 & 48 & 12 & 4 & 74 \\
FIE & 13 & 28 & 4 & 0 & 45 \\
MECANICA & 7 & 45 & 11 & 0 & 63 \\
CIENCIAS & 3 & 55 & 8 & 1 & 67 \\
RECURSOS & & & & & \\
NATURALES & 4 & 22 & 7 & 0 & 33 \\
SALUD PUBLICA & 14 & 34 & 16 & 0 & 64 \\
CIENCIAS & 2 & 21 & 5 & 0 & 28 \\
TOTAL & $\mathbf{5 3}$ & $\mathbf{2 5 3}$ & $\mathbf{6 3}$ & $\mathbf{5}$ & $\mathbf{3 7 4}$ \\
PORCENTAJE & $14 \%$ & $68 \%$ & $17 \%$ & $1 \%$ & $100 \%$ \\
\hline
\end{tabular}

El $68 \%$ de estudiantes dan una calificación regular acerca de la movilidad que existe dentro de la ESPOCH. El 17\% de la población califican como mala la movilidad interna del establecimiento. El 14\% de encuestados dan como resultado que existe una buena movilidad interna en el campus universitario. El 1\% de las personas desconocen el tema de movilidad.

Tabla No19. Cree usted que la señalética de tránsito que existe en la ESPOCH, mejoraría la movilidad

\begin{tabular}{lrrrr}
\hline \multicolumn{1}{c}{ FACULTAD } & SI & \multicolumn{2}{c}{ NO } & \multicolumn{2}{c}{ S/N } & \multicolumn{2}{c}{ TOTAL } \\
\hline FADE & 52 & 19 & 3 & 74 \\
FIE & 27 & 18 & 0 & 45 \\
MECANICA & 49 & 14 & 0 & 63 \\
CIENCIAS & 37 & 28 & 2 & 67 \\
RECURSOS & & & & \\
NATURALES & 26 & 7 & 0 & 33 \\
SALUD PUBLICA & 41 & 23 & 0 & 64 \\
CIENCIAS & 18 & 10 & 0 & 28 \\
TOTAL & $\mathbf{2 5 0}$ & $\mathbf{1 1 9}$ & $\mathbf{5}$ & $\mathbf{3 7 4}$ \\
PORCENTAJE & $67 \%$ & $32 \%$ & $1 \%$ & $100 \%$ \\
\hline
\end{tabular}

El 67\% de la población encuestada piensan que con el incremento de señalética mejoraría su movilidad dentro del establecimiento. El 32\% piensa que al aumentar la señalética no beneficiaria en nada la movilidad que existe dentro de la institución. El 1\% se reserva sus comentarios. 
Gráfico No3. Los parqueaderos de autos y bicicletas se encuentran en buen estado y son próximo a su lugar de destino
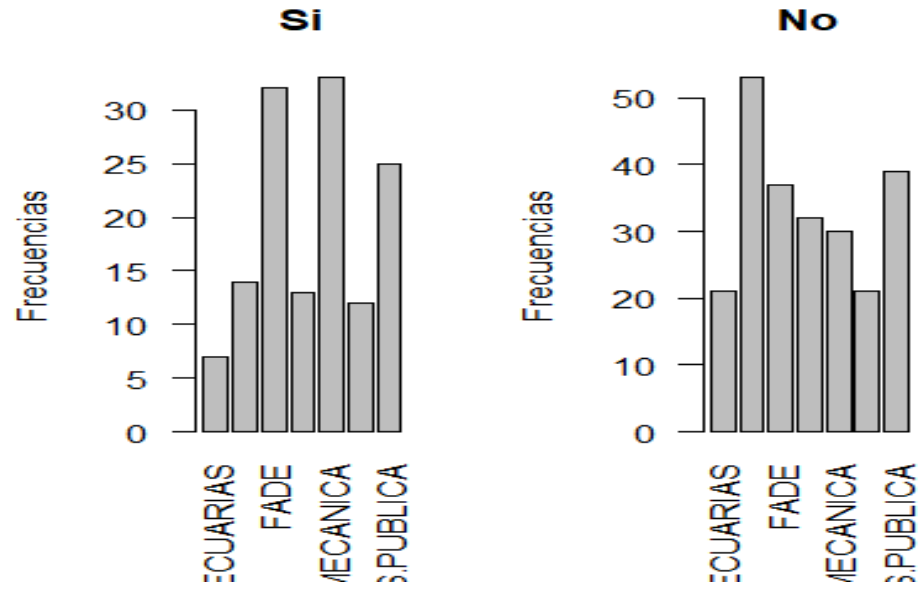

El $62 \%$ de estudiantes recalcan que los parqueaderos de autos y bicicletas no se encuentran en buen estado y tampoco próximos a su lugar de destino. El $36 \%$ de encuestados reflejan estar de acuerdo que están en buen estado los parqueaderos que existen en la institución. El 1\% desconoce el tema tratado.

\section{Discusión y Resultados}

Las ciudades europeas también han realizado esfuerzos para evitar el congestionamiento del tránsito en las áreas centrales de las ciudades o en las cercanías de las grandes zonas atractores de viajes. Debido al crecimiento de la matrícula estudiantil y el fortalecimiento del sistema de educación superior, las universidades han seguido los lineamientos de la Comunidad Europea para contribuir a la movilidad sostenible, en términos de reducir la contaminación provocada por los vehículos automotores, utilizar modos alternativos y promover el transporte multimodal. (Cordero, 2014)

En la ESPOCH se debe optar por modelos parecidos a estos, es decir aplicar la movilidad sostenible, pues el crecimiento estudiantil en la politécnica a crecido paulatinamente y por ende el flujo vehicular es abrumador en horas pico.

En Estados Unidos los modos peatonal y ciclista son los más comunes dentro del perímetro de los campus. La mayoría de las universidades intentan desarrollar un ambiente seguro para peatones y ciclistas. Incluso algunas instituciones en sus planes maestros colocan a los peatones, bicicletas y transporte público como los modos prioritarios en el campus. Las mejores prácticas se organizan en tres grupos que son infraestructura, incentivos y seguridad. (Cordero, 2014)

En el campus de la ESPOCH como se vio en los resultados si hace falta un mejor plan de movilidad que involucre un numero adecuado de paradas, vías y aceras bien delimitadas, y un ciclo vial total con esto se evitara que circulen vehículos particulares dentro del campus y se haría uso solo del trasporte politécnico y la utilización de transporte no motorizado obteniendo ambientes seguros y saludables.

Por otra parte el Departamento de Movilidad de la ESPOCH debe contemplar dentro de sus actividades la planificación de capacitaciones periódicas a todo el personal de universidad con el propósito de conocer señalética, movilidad y Educación Vial, aspectos importantes para obtener una movilidad adecuada y sostenible. 


\section{Conclusiones.}

- No existe un control adecuado de ingreso de vehículos particulares y comerciales al momento que ingresan a la Escuela Superior Politécnica de Chimborazo.

- Se puede reflejar la falta de una señalética adecuada dentro del campus universitario.

- Existe evidencia estadística que gran parte de los estudiantes no utilizan el servicio de transporte de la ESPOCH porque sus frecuencias no son adecuadas.

- Un buen número de encuestados reflejan su desconocimiento de lo que es Movilidad y Educación Vial.

\section{Referencias bibliográficas}

Castro García, L. J. (2012). ESTRATEGIAS DE MOVILIDAD SOSTENIBLE PARA FORTALECER LA RESPONSABILIDAD CORPORATIVA EN EMPRESAS . Quito: Ecuarom.

(20 de Agosto de 2015). Obtenido de German Partenership For Mobility, Sustainable: https://www.german-sustainable-mobility.de/wpcontent/uploads/2016/04/GPSM_About-Us_Spanish_web.pdf

Cordero, L. F. (2014). La movilidad sostenible en campus universitarios: una comparación de las mejores prácticas en Estados Unidos y Europa. Aplicabilidad en universidades venezolanas. Scielo, 12.

Escobar, D. A. (2018). Gestion del Territorio e Infraestructura. Barcelona: Alban.

Mercham, M. E. (2011). Seguridad Vial y Peatonal: Una aproximacion teorica desde la politica publica. Scielo, 15.

Minta, A. (2016). PLAN INTEGRAL DE EDUCACIÓN VIAL. Riobamba.

Montes de Oca, N., \& Machado, R. (2009). El desarrollo de habilidades investigativas en la educación superior: un acercamiento para su desarrollo. Humanidades Médicas, 9(1), 415. Obtenido de http://scielo.sld.cu/scielo.php?script=sciarttext\&pid=S1727$81202009000100003 \& \ln \mathrm{g}=\mathrm{es} \& \ln \mathrm{g}=\mathrm{es}$

OMS. (2018). Obtenido de https://www.eltiempo.com/vida/salud/informe-de-la-oms-sobreaccidentes-de-transito-en-el-mundo-302888

OMS. (2018). Obtenido de https://latinamericanpost.com/es/18978-accidentes-viales-enamerica-latina

Rosero, M. A., \& Romero, E. S. (2012). Movilidad Sostenible para Fortalezer la responsabilidad de las Empresas. Quito.

Ruiz, R. (27 de febrero de 2007). Metodo Cientifico y sus etapas. Obtenido de http://www.index-f.com/lascasas/documentos/lc0256.pdf

Sarahi, A. (2005). Fórmulas para el cálculo de la muestra en investigaciones de salud. Redalyc, 7. 
Sarai, A. (2005). Fórmulas para el cálculo de la muestra en investigaciones de salud. Redalyc, 7.

Urbano, P. M. (20 de 12 de 2012). B- Digital. Obtenido de https://revistas.unal.edu.co

Villarroel, G. (26 de 01 de 2018). Análisis de la calidad de servicio del transporte público urbano y su mejora continua a través de la Norma UNE-EN 13816 en el cantón Pastaza, provincia de Pastaza. Obtenido de DSpace: http://dspace.espoch.edu.ec/handle/123456789/8422 


\section{Para citar el artículo indexado.}

Londo Yachambáy, F. P., \& Sánchez Salcán, N. de J. (2019). Análisis Estadístico de Movilidad y Educación Vial dentro de la Escuela Superior Politécnica de Chimborazo. Explorador Digital, 3(3), 90-103. https://doi.org/10.33262/exploradordigital.v3i3.764

\section{Ciencia \\ LDigital}

El artículo que se publica es de exclusiva responsabilidad de los autores y no necesariamente reflejan el pensamiento de la Revista Explorador Digital.

El articulo queda en propiedad de la revista y, por tanto, su publicación parcial y/o total en otro medio tiene que ser autorizado por el director o editor de la Revista Explorador

Digital.
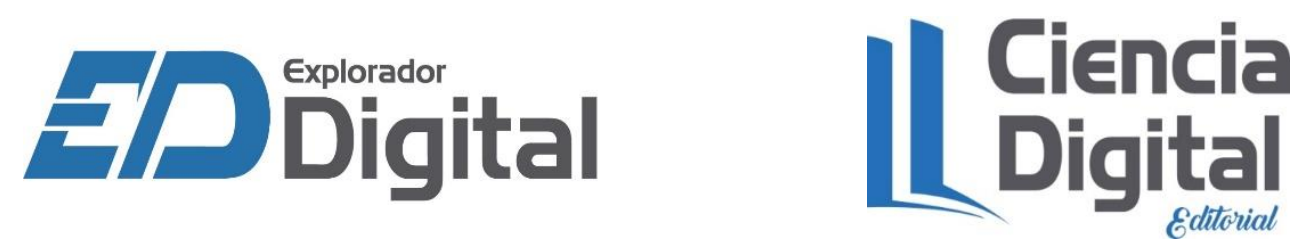